\title{
Performance and applications of a 100-element HBT grid amplifier
}

\author{
David B. Rutledge, Mconil Kim ${ }^{1}$, Emilio A. Sovero, ${ }^{2}$ Michael P. De Lisio, Jonathan B. Hacker, \\ Jung-Chih Chiao, Shi-Jie Li, David R. Gagnon, ${ }^{3}$ and James J. Rosenberg, \\ Division of Engineering and Applied Science, California Institute of Technology, Pasadena, CA 91125 \\ ${ }^{1}$ Central Research Laboratory, Gold Star, 16 Woomyen-Dong, Seocho-Gu, Seoul 137-40, Korea \\ ${ }^{2}$ Science Center, Rockwell International Corporation, 1049 Camino Dos Rios, Thousand Oaks, CA 91385 \\ ${ }^{3}$ Naval Air Warfare Weapons Division, Naval Weapons Center, China Lake, CA 93555-6001 \\ ${ }^{4}$ Engineering Department, Harvey Mudd College, Claremont, CA 91711
}

\begin{abstract}
A 100-element 10-GHz grid amplifier has been developed. The active devices in the grid are chips with heterojunction bipolar transistor (HBT) differential pairs that include a resistive network to provide self-bias to the base. The planar metal grid structure was empirically designed to provide effective coupling between the HBT's and free space. The peak gain of the grid was $10 \mathrm{~dB}$ at $10 \mathrm{GHz}$ with a $3 \mathrm{~dB}$ bandwidth of $1 \mathrm{GHz}$. The input and output matches are better than $15 \mathrm{~dB}$ at $10 \mathrm{GHz}$. The maximum output power is $450 \mathrm{~mW}$, and the minimum noise figure is $7 \mathrm{~dB}$. Tests show that the grid is quite tolerant of failures-the output power dropped by only $1 \mathrm{~dB}$ when the $10 \%$ of the inputs were detuned. The device amplifies beams with incidence angles up to $30^{\circ}$ with less than a 3-dB drop in power. By providing external feedback with a twist reflector, the grid amplifier can be used as a broadband tunable source. This source could be tuned from $6.5 \mathrm{GHz}$ to $11.5 \mathrm{GHz}$ with a peak effective radiated power (ERP) of $6.3 \mathrm{~W}$ at $9.9 \mathrm{GHz}$.
\end{abstract}

\section{INTRODUCTION}

Quasi-optical grids offer the possibility of large-scale power combining of solid-state devices without the limitations of transmission-line combiners. A 25-element MESFET grid amplifier [1] was demonstrated that amplifies a beam, with cross-polarized input and output (Figure 1). This configuration suppresses spurious oscillations, and allows polarizers to tune the input and output independently. This first grid had metal strips on the back for biasing, and resistors and pins to connect to packaged transistors on the front. Recently a 16-element planar grid with custom differential-pair heterojunctionbipolar-transistor (HBT) chips was demonstrated, with a gain of $11 \mathrm{~dB}$ at $10 \mathrm{GHz}$ [2]. However, the grid was small, little more than a wavelength across, making other measurements difficult. Here we report a much larger $100-$ element grid amplifier that is nearly three wavelengths

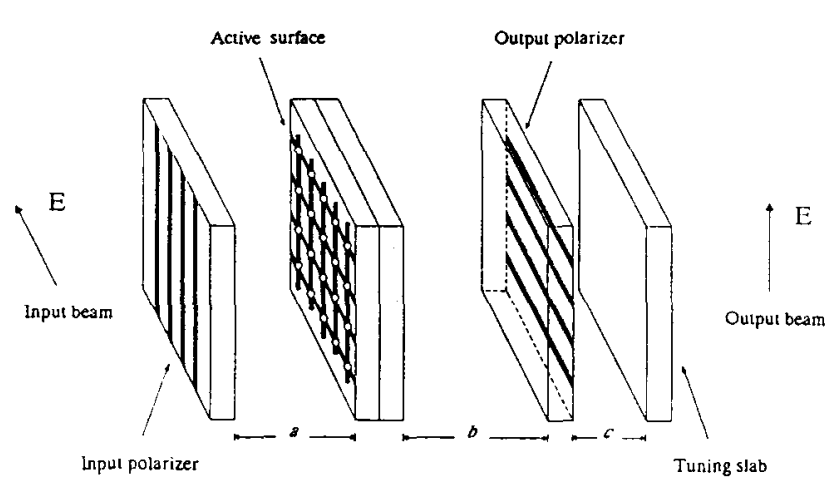

Figure 1. A perspective view of a grid amplifier. The input beam is horizontally polarized and the output beam is vertically polarized.

across. We have made measurements of gain, input and output match, saturated power, noise figure, reliability, and angled beams. In addition, we have used the grid amplifier to make a broadband tunable oscillator by providing external feedback with a twist reflector.

\section{FABRICATION}

The differential-pair chips contain two HBT's along with a simple bias network to provide self-bias to the base as shown in Figure 2a. The values of the resistors, $1.7 \mathrm{k} \Omega$ and $500 \Omega$, were picked under the assumption that $\beta=$ 10 , to set $V_{c e}=3 \mathrm{~V}$ and $I_{c}=10 \mathrm{~mA}$. This bias network eliminates the need for an extra base-bias line on the grid that would complicate the metal pattern. In addition, these resistors also help to suppress spurious oscillations. The $1.7-\mathrm{k} \Omega$ collector-to-base resistor provides negative shunt feedback, and the $500-\Omega$ emitter resistor reduces the common-mode gain of the HBT pair. This prevents the structure from acting like a grid oscillator [3].

Many different metal patterns for the grid amplifiers were tested on smaller $4 \times 4$ grid arrays. Best results were obtained from a grid with the unit-cell dimensions shown in Figure 2b. This designewas then adopted for a $10 \times 10$ grid. In the design, $100 \mu \mathrm{m}$ wide meander lines provide bias to the HBT's. Ideally, the bias lines should present 


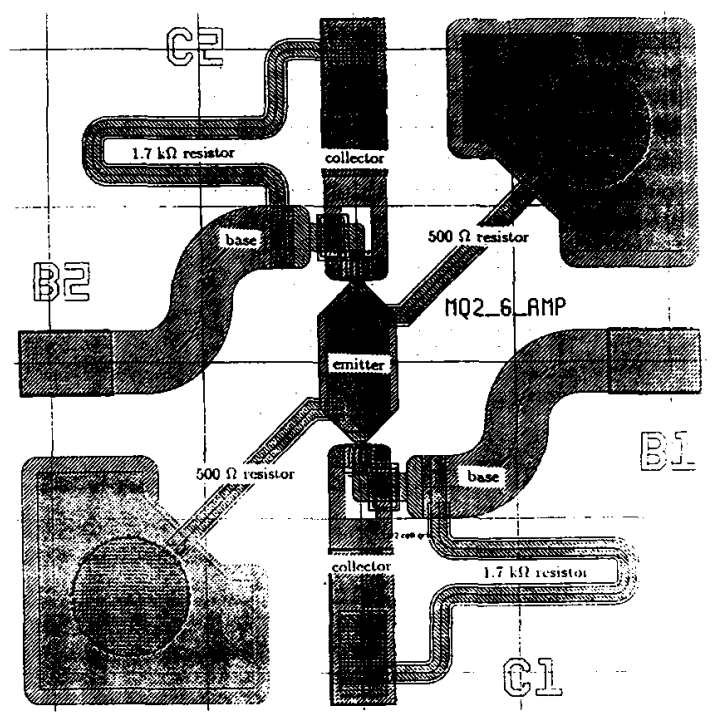

(a)

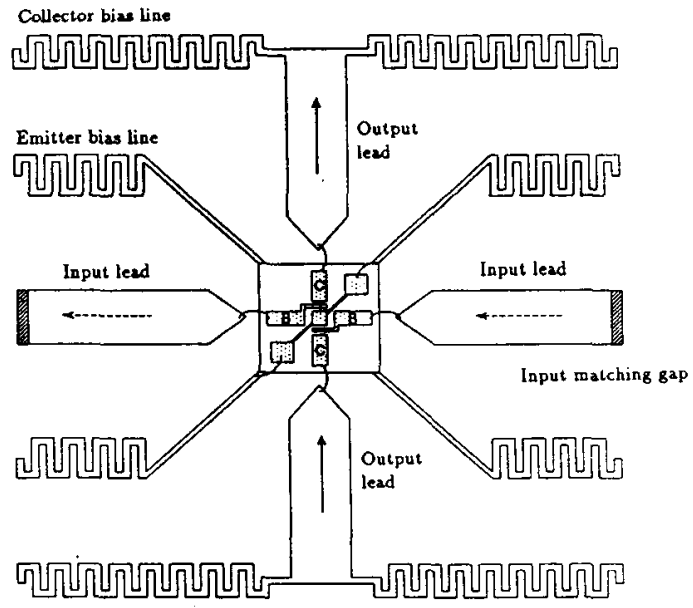

(b)

Figure 2. (a) The differential-pair HBT chip layout. 1.7$\mathrm{k} \Omega$ and $500-\Omega$ resistors provide self-bias to the base. The chip requires dc bias connections only to the collector and the emitter. (b) Sketch of a unit cell. The unit cell size is $8 \mathrm{~mm}$. The radiating leads are $0.8 \mathrm{~mm}$ wide while the bias lines are only $100 \mu \mathrm{m}$ wide. The extremes of the meander bias lines span $0.6 \mathrm{~mm}$ from top to bottom and have gaps $100 \mu \mathrm{m}$ wide. The gap in the input antenna lead is $100 \mu \mathrm{m}$ wide.

a large reactance, so that they do not disturb incident and radiated fields. A $100 \mu \mathrm{m}$-wide gap is made in the input leads to add a series capacitance to compensate for their large inductive reactance.

\section{Amplifier Measurements}

A lens-focused microwave beam was used to measure the gain and reflection coefficients of the grid amplifier [4]. During the measurements, a bias of $5 \mathrm{~V}$ and $860 \mathrm{~mA}$ was applied to the entire grid. For the individual transistors,

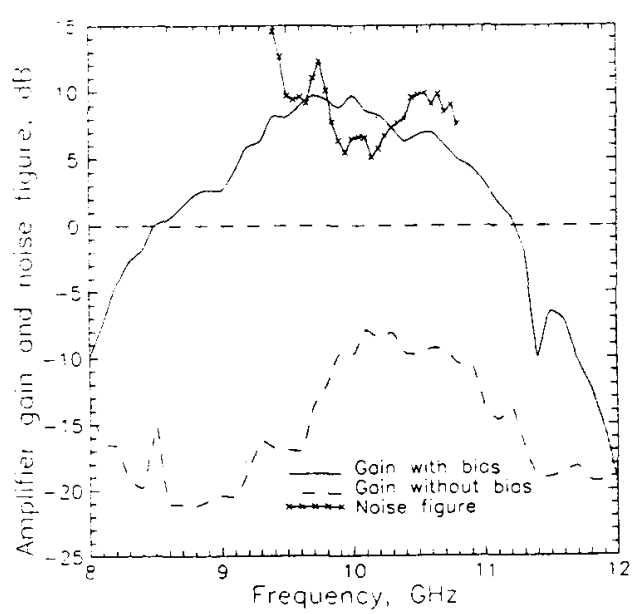

Figure 3. The grid amplifier gain and noise figure.

we estimate that $V_{c e}=2.9 \mathrm{~V}, I_{c}=3.4 \mathrm{~mA}$, and $\beta=3.7$. The positions of the polarizers were carefully adjusted to produce the largest amplifier gain. The results show that the peak gain was $10 \mathrm{~dB}$ at $10 \mathrm{GHz}$ with a $3-\mathrm{dB}$ bandwidth of $1-\mathrm{GHz}$ (Figure 3). The lens system was also used as a reflectometer to measure the input and output reflection coefficients. The results indicate that the input and output return loss is better than $15 \mathrm{~dB}$ at $10 \mathrm{GHz}$.

The gain measurement was repeated using a horn source in the far field of the grid [1], with similar results to those measured with lenses. In addition, the grid amplifier noise performance was also measured for the first time. Using a Hewlett Packard HP8970 Noise Figure Meter and Watkins Johnson M17C X-band mixer, the noise figure of the whole measurement setup including the two horns and the grid was measured. A noise diode with a high excess noise ratio (ENR) of about $40 \mathrm{~dB}$ was required. We used a Noise-Com NC3206. The noise figure of the grid amplifier could then be extracted from the measured noise figure, and is plotted in Figure 3. The minimum noise figure for a $5-\mathrm{V}$ bias fluctuated between $7 \mathrm{~dB}$ and $8 \mathrm{~dB}$ in the frequency range from 10 to $11 \mathrm{GHz}$. As the bias was lowered from 5 to $4 \mathrm{~V}$, the noise figure decreased by about $1 \mathrm{~dB}$, but the amplifier gain dropped by $1 \mathrm{~dB}$ as well. This noise figure compares to the $4-\mathrm{dB}$ optimum noise figure previously reported for single IIBT's [5].

The grid saturation characteristics were also measured with the iar-field horn source, so that the input beam had a uniform amplitude and phase across the grid. The 1- $\mathrm{dB}$ gain compression point was $105 \mathrm{~mW}$, and the saturated output power was $450 \mathrm{~mW}$. The overall dc power supplied to the grid was $4.3 \mathrm{~W}$, with power divided equally between the bias resistors and the transistors. The maximum power-added-efficiency, at an output power level of $330 \mathrm{~mW}$, was $5 \%$, with the loss in the bias resistors included. 


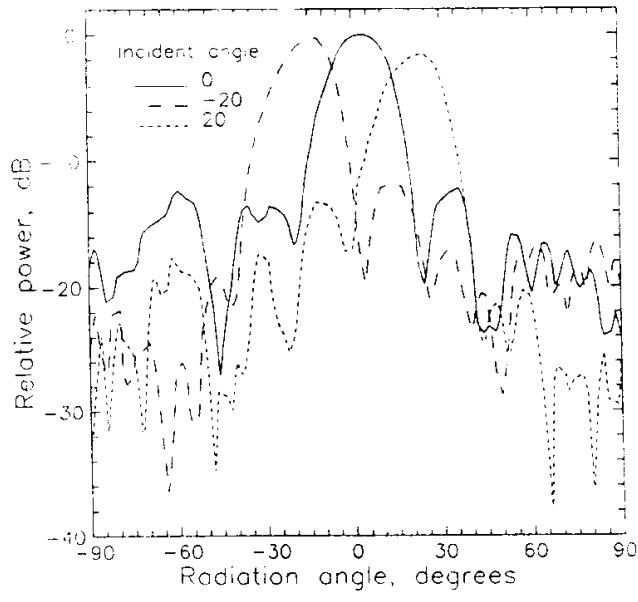

(a)

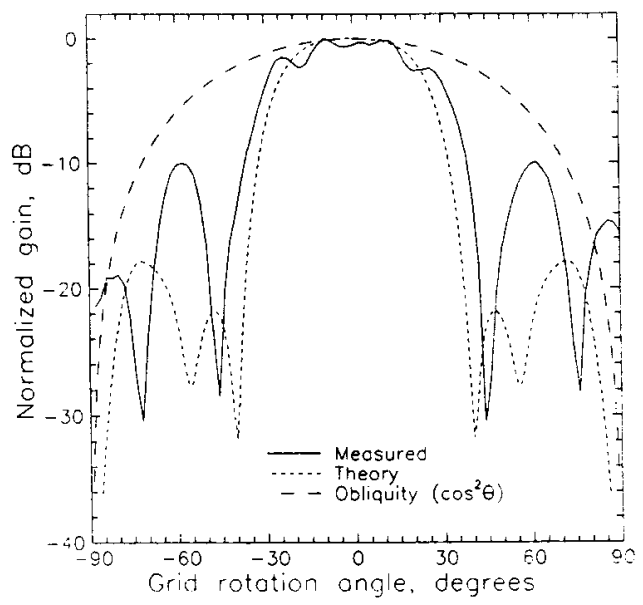

(b)

Figure 4. The grid amplifier radiation patterns for different incident beam angles (a). The transmission loss as the grid amplifier rotates (b). This shows how the amplitude changes as the angle of incidence varies. Also plotted are the theoretical transmission loss and the $\cos ^{2} \theta$ obliquity factor.

The effect of failures in the grid was simulated by connecting bond wires across the gaps in the input leads. This severely detunes the inputs. We found that with $10 \%$ of the gaps shorted, the output field dropped by $10 \%$, so that the power fell by $1 \mathrm{~dB}$. With a quarter of the gaps shorted, the output power dropped by $5 \mathrm{~dB}$. With half of the gaps shorted, the output power dropped all the way down to the zero-bias level.

A grid amplifier is a multi-mode device that should preserve the propagation angles of incident beams. To test this idea, the beam patterns were measured with input plane waves at normal incidence and $20^{\circ}$ off axis. Figure $4 \mathrm{a}$ shows the resulting three output beams. The beamwidths and sidelobe levels for all beams are those that we would expect for plane-wave illumination, but the angles shift to match the input beams. The angle error is about $1^{\circ}$. To test the amplitude variation, a grid amplifier was rotated between two far-field horns. The roll-off is less than $3 \mathrm{~dB}$ for angles less than $30^{\circ}$, as shown in Figure $4 \mathrm{~b}$.

The theoretical curve in Figure $4 \mathrm{~b}$ was generated using simple transmission-line models for the air-gaps, polarizers, and dielectric tuning slabs in the grid amplifier. The impedance representing the active surface of the amplifier was chosen so that the grid was input and output matched at normal incidence. The transmission loss of the amplifier could then be computed as a function of incidence angle by scaling all of the transmission line lengths by $\cos \theta_{i}$ and the characteristic impedances of the lines by $\cos \theta_{i}$ (TM polarization) or $\sec \theta_{i}$ (TE polar ization), where $\theta_{i}$ is incidence angle in the appropriate media. The entire pattern is then scaled by a $\cos ^{2} \theta$ obliquity factor to account for the foreshortening of the grid. Theory agrees well with experiment for small angles of incidence. The nulls in the pattern result when the effective distance between a polarizer and the active surface of the grid is such to present a short circuit to the grid. By making a more compact grid, the nulls could conceivably be eliminated, and the pattern would approach that of the obliquity factor alone.

\section{Oscillator MEasurements}

The grid amplifier can be used to make a tuneable oscillator by replacing the input polarizer with a twist reflector, as shown in Figure 5. The twist reflector consists of a metal strip polarizer backed by a mirror [6]. The strips of the polarizer are oriented at a $45^{\circ}$ angle to the electric field of a normally incident plane wave. The mirror is positioned a quarter-wavelength behind the polarizer so the reflected wave will be polarized orthogonal to the incident wave. This provides the necessary feedback between the orthogonally polarized output and input of the grid amplifier.

The oscillation frequency could be tuned smoothly between $8.2 \mathrm{GHz}$ and $11.0 \mathrm{GHz}$ by changing the spacing of the grid amplifier and the twist reflector (Figure 6). The effective radiated power (ERP) varied from $-5 \mathrm{dBW}$ to $8 \mathrm{dBw}$ (Figure 6). The frequency and ERP show a halfwavelength period from higher-order longitudinal modes. The maximum ERP was $6.3 \mathrm{~W}$ at $9.9 \mathrm{GHz}$. By changing both the separation of the mirror and the $45^{\circ}$ polarizer that compose the twist reflector (dimension $a$ in Figure 5) and the spacing between the grid amplifier and the twist reflector, the oscillator could be tuned from $6.5 \mathrm{GHz}$ to 11.5 GHz - a 1.8-to-1 bandwidth.

\section{CONCLUSion}

We have presented a 100-element grid amplifier with a gain of $10 \mathrm{~dB}$ at $10 \mathrm{GHz}$, a $10 \% 3-\mathrm{dB}$ bandwidth, and an 


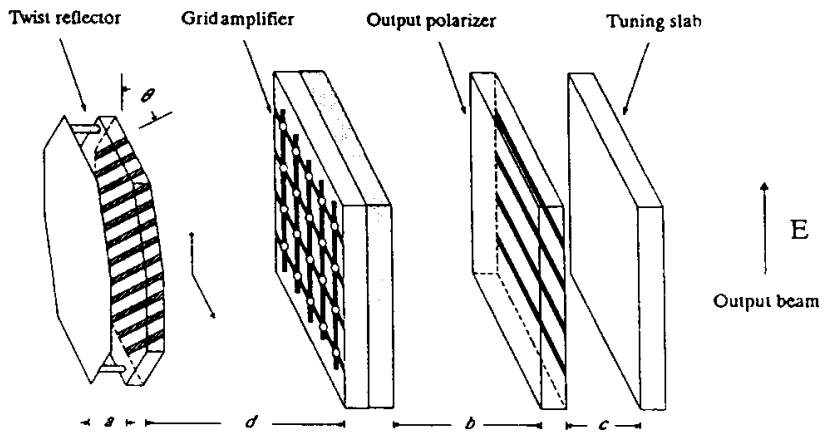

Figure 5. The external feedback oscillator. The twist reflector that provides the feedback replaces the input polarizer in the normal grid amplifier.

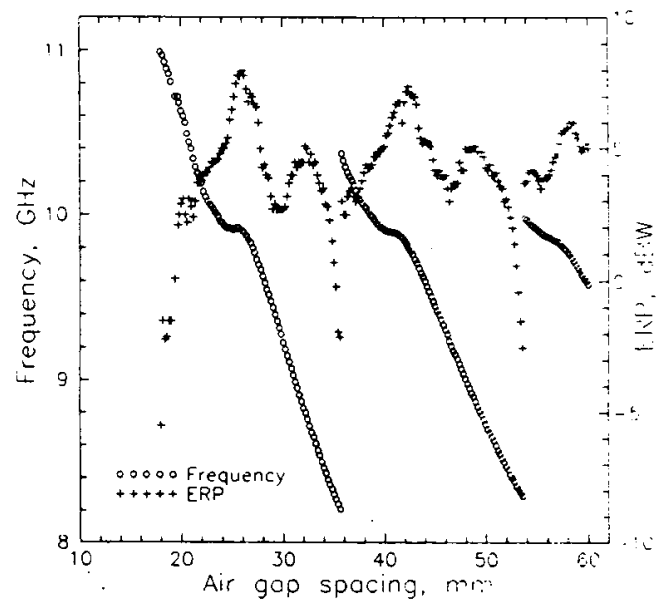

Figure 6. Tuning the oscillator by changing the distance between the grid amplifier and the twist reflector (dimension $d$ in Figure 5).

input and output match better than $15 \mathrm{~dB}$. The maximum output power is $450 \mathrm{~mW}$ and the minimum noise figure is $7 \mathrm{~dB}$. The results are consistent with the idea that the gain, bandwidth, input and output match, and noise figure should be comparable to single transistor amplifiers, but that the power scales with the size of the grid. The grid degrades gracefully $-10 \%$ failures produce only a $1-\mathrm{dB}$ drop in gain. This means that a grid amplifier could be much more reliable than a single high-power solid-state device or vacuum tube. The grid amplifies beams at incident angles up to $30^{\circ}$. This means that a grid amplifier could amplify a beam for an electronicbeam steerer, easing the loss requirements for the steering system. Furthermore, we have shown that when used with a twist reflector, this grid amplifier could be used as a $6.5 \mathrm{GHz}$ to $11.5 \mathrm{GHz}$ tunable source with a peak ERP of $6.3 \mathrm{~W}$ at $9.9 \mathrm{GHz}$.
More details of this work may be found in the upcoming special issue on Quasi-Optical Techniques of the IEEE Transactions on Microwave Theory and Techniques, October, $1993[7,8]$.

\section{ACKNOWLEDGEMENTS}

The work at Caltech is supported by the Army Research Office, the Northrop Corporation, the Naval Weapons Center in China Lake, CA, and a fellowship from the Rockwell International Trust.

\section{REFERENCES}

[1] M. Kim, J.J. Rosenberg, R.P. Smith, R.M. Weikle, J.B. Hacker, M.P. De Lisio, D.B. Rutledge , "A Grid Amplifier," IEEE Microwave and Guided Wave Letters, MGWL-1, pp. 322-324, May 1991.

[2] D.B. Rutledge, J.B. Hacker, M. Kim, R.M. Weikle, R.P. Smith, E.A. Sovero, "Oscillator and Amplifier Grids," IEEE MTT Int.Symp., Albuquerque, NM, pp. 815-817, June 1991.

[3] R.M. Weikle, M. Kim, J.B. Hacker, M.P. De Lisio, D.B. Rutledge, "Planar MESFET Grid Oscillators using Gate Feedback," IEEE Trans. Microwave Theory Tech. MTT-40, pp. 1997-2003, November 1992.

[4] D.R. Gagnon, "Highly Sensitive Measurements with a Lens-Focused Reflectometer," IEEE Trans. Microwave Theory Tech. MTT-39, pp. 2237-2240, December 1991 .

[5] P.M. Asbeck, M-C.F. Chang, J.A. Higgins, N.H. Sheng, G.J. Sullivan, K.C. Wang, "GaAs/AlGaAs Heterojunction Bipolar Transistors: Issues and Prospects for Application," IEEE Trans. Electron Devices, ED-36, pp. 2032-2035, October 1989.

[6] Microwave Antenna Theory and Design, edited by Samuel Silver, volume 12 in the MIT Radiation Laboratory Series, published by McGraw-Hill in 1949. Republished in 1984 by Peter Peregrinus Ltd. pp. 447.

[7] M. Kim, E.A. Sovero, J.B. Hacker, M.P. De Lisio, J-C. Chiao, S-J. Li, D.R. Gagnon, J.J. Rosenberg, and D.B. Rutledge, "A 100-Element HBT Grid Amplifier," to be published in IEEE Transactions on Microwave Theory and Techniques, October, 1993.

[8] M. Kim. E.A. Sovero, J.B. Hacker, M.P. De Lisio, J.J. Rosenberg, and D.B. Rutledge, "A $6.5 \mathrm{GHz}$ $11.5 \mathrm{GHz}$ Source Using a Grid Amplifier with a Twist Reflector." to be published in IEEE Transactions on Microwave Theory and Techniques, October, 1993. 